\title{
A New Automated Trigger Circuit for a Pulsed Nd: YAG Laser
}

\author{
Fatah_Almabouada, Djelloul Louhibi, Abderrahmane Haddouche, \\ Abdelkader Noukaz and Ramdan Beggar \\ Centre de Développement des Technologies Avancées, \\ Division des Milieux Ionisés \& Laser \\ BP 17, Cité du 20 août 1956, Baba Hassen, Algiers, Algeria \\ Email: falmabouada@yahoo.fr
}

\begin{abstract}
The power supply of flash lamp-pumped a Nd: YAG solid-state laser basically consists of a trigger circuit which initiate the ionization of the gas into the lamp, a simmer circuit that keeps the ionized gas with a low-level dc current and a main discharge circuit where a storage capacitor is discharged into a flash lamp to pump the Nd:YAG laser. The impedance of the flash lamp presents a nonlinear variation and the gas deionization which can occur between two pulses laser, leading to another manual triggering operation. To overcome this operation, we give in this paper a new automated trigger circuit activated by a delivered signal from the simmer circuit. This circuit is simple for implementation and need just few components. It has been simulated by PSpice software and validated by experimental results.
\end{abstract}

Keywords: Flash lamp, solid-state laser, simmer circuit, trigger circuit.

\section{Introduction}

Pulsed Xenon flash lamps are gas-discharge devices designed to produce a pulsed radiation. They convert the electrical energy to the optical radiation. This intense pulse of radiant energy is used in many applications. In our case these devices are used for pumping solid-state lasers.

One of the principle diagrams of the power supply circuit of a pulsed flash lamp is the simmer mode operation as shown in Fig. 1. This mode has a several advantages and one of them is the increasing of the flash lamp lifetime.

The power supply performs a number of functions:

- The external trigger circuit provides a high voltage pulse around $20 \mathrm{kV}$ to initiate the ionization of the gas. The secondary of the trigger transformer is connected to a wire which is wrapped around the lamp [1]-[2].

- The simmer circuit maintains a partial steady-state ionization by establishing and maintaining a low-current dc arc between the lamp electrodes.

- The charging unit is used to charge the storage capacitor $\mathrm{C}$. The charging must be completed within the inter-pulse time of the laser. The principal capacitor discharge through the flash is ensured by an electronic switch. The capacitor $\mathrm{C}$, the inductor $\mathrm{L}$ 
and the impedance $\mathrm{R}$ of the flash lamp constitute the circuit that provides the desired shape pulse during the discharge. The operation of the laser and the flash lamp characteristic, allow to fix the values of $\mathrm{C}$ and $\mathrm{L}$ [2].

During the operation of the laser, the lamp gas can be deionized between two laser pulses obliging to trigger again which is a drawback because in our previous work the triggering operation was activated by a manual command.

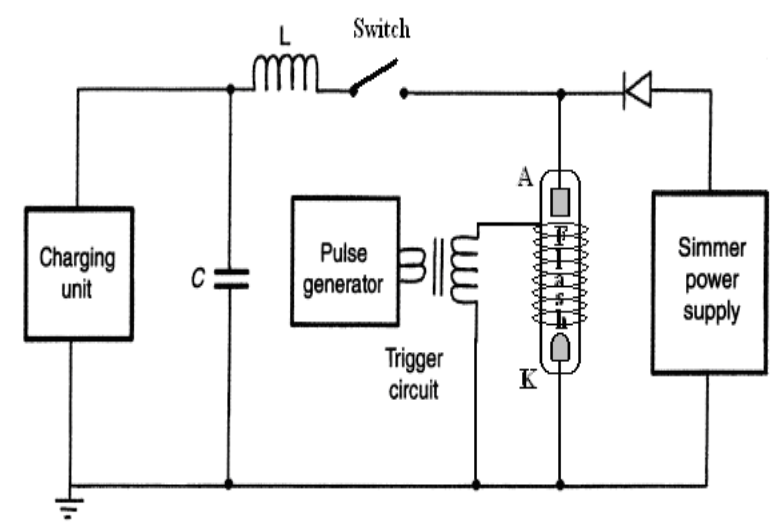

Fig. 1. Principle diagram of the flash tube excitation circuit.

\section{Contribution to Technological Innovation}

This work is an innovation in the electronic command of a trigger circuit used to energize the flash lamp. In our contribution the command trigger is automated by a circuit which isn't complex and uses little components. Several works were done in this field [3] and where the trigger is activated at each laser pulse which is a disadvantage because that decreases the flash lamp lifetime [2].

\section{Simmer and Trigger Circuit}

\subsection{Simmer Circuit}

After the triggering, the flash lamp impedance $\mathrm{R}$ varies in a nonlinear way where its measured value is given within $1 \mathrm{k} \Omega$ and $17 \mathrm{k} \Omega$ [4]. This variation is represented by the electrical characteristic of specified flash lamp as illustrated in Fig. 2. 


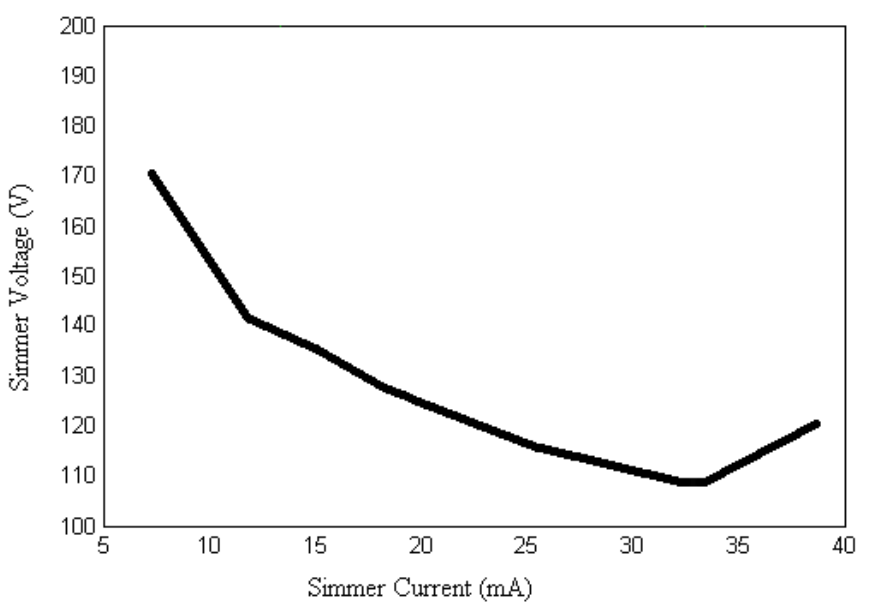

Fig. 2. Electrical characteristic of the flash lamp (Flash-Heraeus Noblelight Ltd. Model: P537).

The simmer circuit will be able to follow this variation. The proposed simmer uses a voltage multiplier which is supplied by $200 \mathrm{~V}$ as an alternative voltage given by a switch mode power supply. This voltage multiplier delivers $1.2 \mathrm{kV}$ and $100 \mathrm{~mA}$ when a flash lamp is non-ignited. At the ionized state, these values vary in accordance with the flash lamp resistor load [5].

Before the experimental step, all the simulated results of designed circuits have simulated using the Pspice simulator.

The simulated simmer circuit is represented in Fig. 3, V1 is equal to $200 \mathrm{~V}$ and the resistor R10 represents the flash lamp resistor taking several values. The simulated results of the simmer voltage are given for several values of R in Fig. 4.

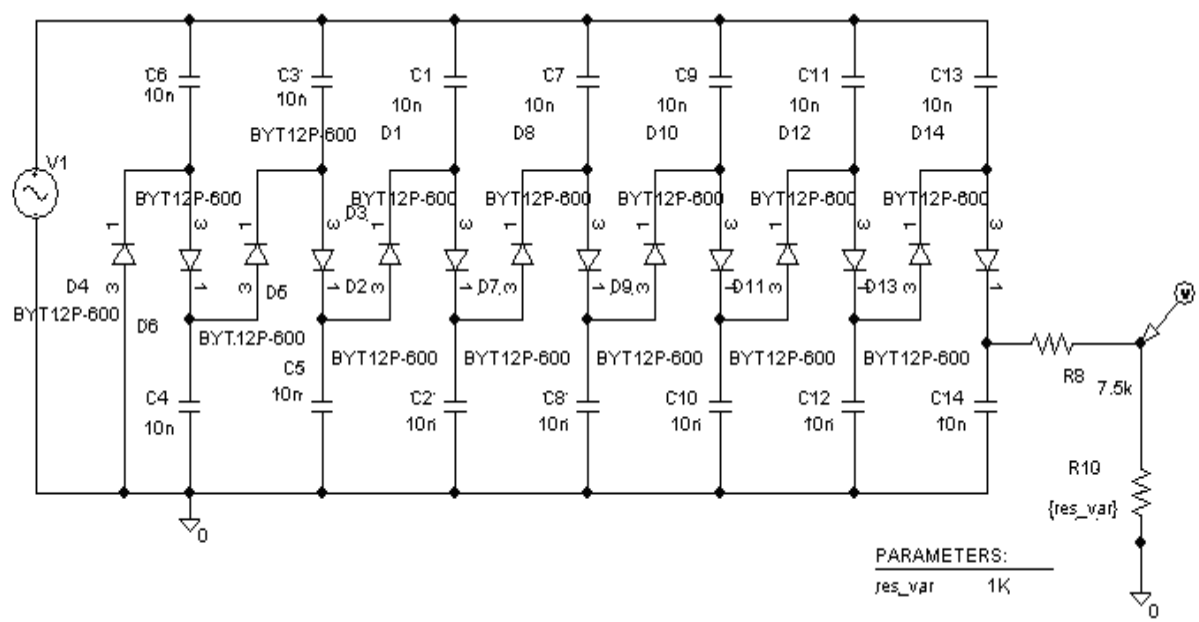

Fig. 3. Simmer circuit. 


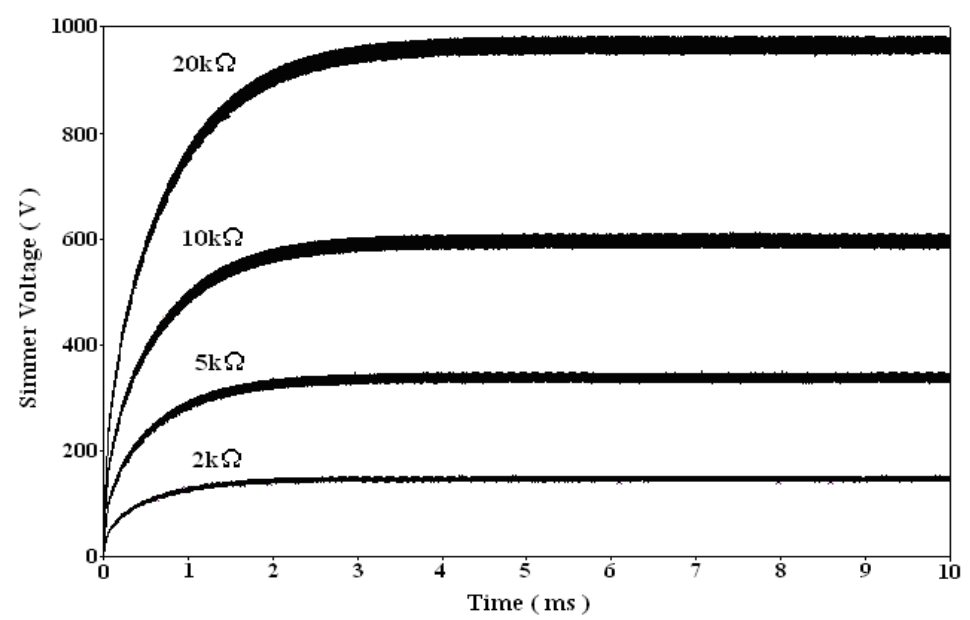

Fig. 4. Variations of the Simmer voltage versus the flash resistor.

\subsection{Trigger Circuit}

In our previous work we developed a trigger circuit activated by a manual command [6]. This trigger (Fig. 5) is a flyback converter [7] using a commercial high voltage transformer (THT model: HR6060). The user has just to push the manual button (S) to supply pulse duration $\mathrm{T}_{1}$ to the switching transistor $(\mathrm{T})$, adjusted by $\mathrm{R} 1$ and $\mathrm{C} 1$ of the monostable MC4538, which will be sufficient to produce the high output voltage $\left(\mathrm{V}_{\mathrm{HT}}\right)$.

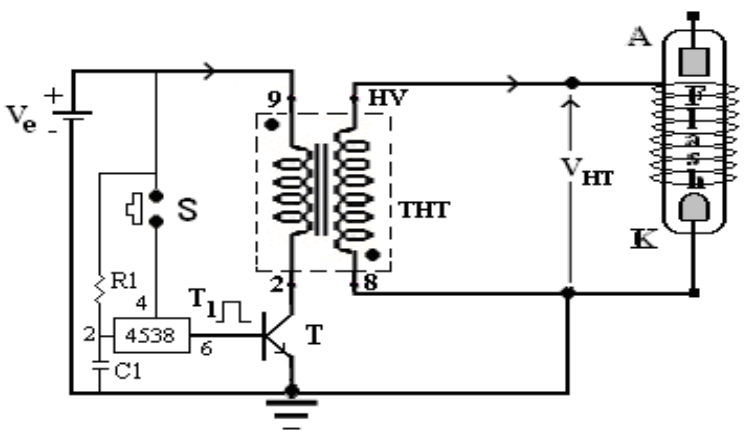

Fig. 5. Trigger circuit.

In general, the THT is supplied by $120 \mathrm{~V}(\mathrm{Ve})$ as a voltage at $15.625 \mathrm{kHz}(64 \mu \mathrm{s})$ allowing pulse duration $T_{1}$ equal to $32 \mu$ s for a duty cycle of $50 \%$. In our case, a low voltage of $12 \mathrm{~V}$ to $24 \mathrm{~V}$ is applied for a safety use; therefore the pulse duration $\mathrm{T}_{1}$ should be adjusted in order to have the same high voltage in the output. For this purpose, the relation between the parameters is expressed by [7]: 


$$
\mathrm{T}_{1}=\left(\mathrm{L}_{\mathrm{p}} / \mathrm{V}_{\mathrm{e}}\right) \mathrm{I}_{\mathrm{pmax}}
$$

where $\mathrm{L}_{\mathrm{P}}$ is the primary inductance and $\mathrm{I}_{\mathrm{Pmax}}$ is the maximum primary current.

For $\mathrm{Ve}=12 \mathrm{~V}$ we must increase $\mathrm{T}_{1}$ at $320 \mu$ s for obtaining the same $\mathrm{I}_{\mathrm{pmax}}$.

The circuit was simulated and tested experimentally. The experimental result is given by Fig. 6 . To avoid the manual triggering after the deionization of the gas, the useful idea is to automate the command of this trigger circuit.

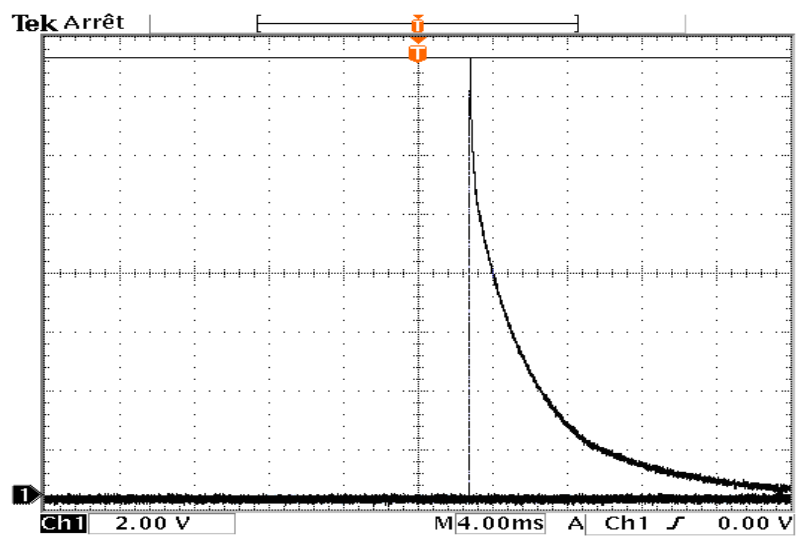

Fig. 6. The triggering pulse (trace vertical: $2 k V / d i v$ )

\subsection{Simmer Command for Triggering}

For the automated command, the command signal is generated by the simmer circuit depending of the ionized state. When the simmer output is around $1.2 \mathrm{kV}$ corresponding to the flash lamp non-ignited state, a pulse voltage is required for the monostable in order to generate the trigger pulse. For the ignited state, the simmer circuit has a small voltage value which is not sufficient to command the monostable. The complete simmer-trigger schematic enclosing the command signal is illustrated in Fig. 7.

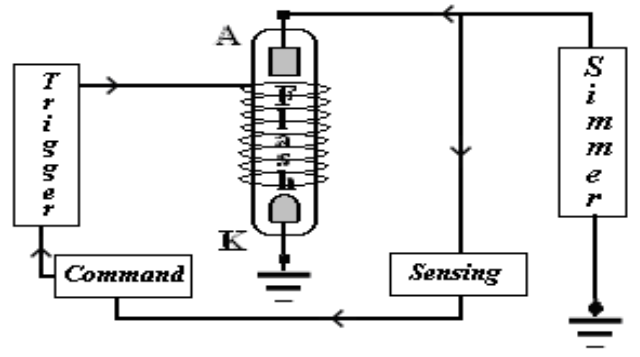

Fig. 7. Simmer-trigger schematic enclosing the command signal. 


\section{Experimental Circuit}

The Fig. 8 shows the experimental circuit.

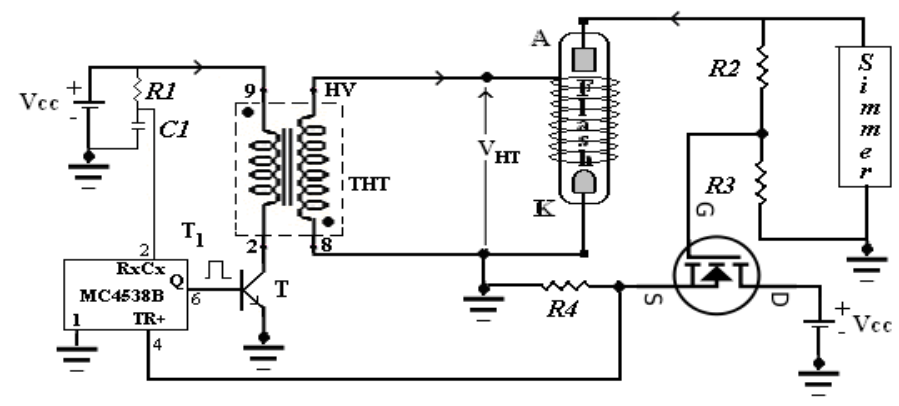

Fig. 8. Experimental circuit of the automated trigger.

Fig. 8 describes the all components to achieve the last reported command function. Furthermore, the Pin 4 of the monostable receives a command signal from the additional circuit used to automate our trigger. This circuit employs a resistor divider and an IGBT (or a MOSFET transistor) as common drain with a simple resistor at its source. For the deionized gas state, the resistor divider (R2, R3) gives a sufficient voltage to the gate of the IGBT which works in the switch-on mode. In this case, the IGBT switches to the minimum required voltage ( $3 \mathrm{~V}$ ) used by the Pin 4 in order to offer the desired $T_{1}$ from the monostable. In the ionized gas state, the resistor divider can not fulfill the voltage condition keeping the IGBT in the switch-off mode.

The simulated results of the monostable input voltage (Pin4) and the simmer voltage verify the behavior of the additional circuit for the automated case as shown in Fig. 9. These results are in good accordance with the experimental results given in the Fig. 10.

Fig. 11 represents the experimental behavior of the simmer voltage and the high voltage triggering pulse. It is observed that when the simmer voltage reaches its high value, the trigger circuit delivers the triggering pulse for gas ionization into the flash lamp. At this moment, the simmer voltage drops down to its small value. The circuit was tested with a Xenon flash lamp: Flash-Heraeus Noblelight Ltd. Model: P537 which is used to pump the Nd: YAG laser. 


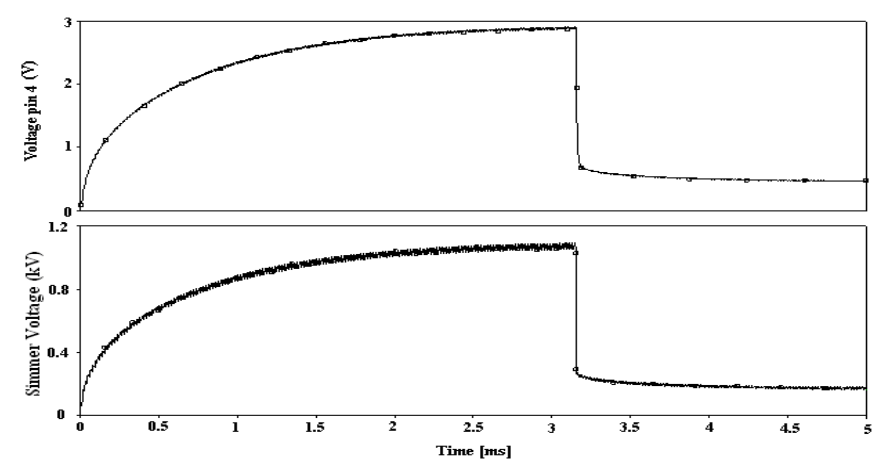

Fig. 9. Simulated results of the simmer voltage and the monostable voltage (pin4).

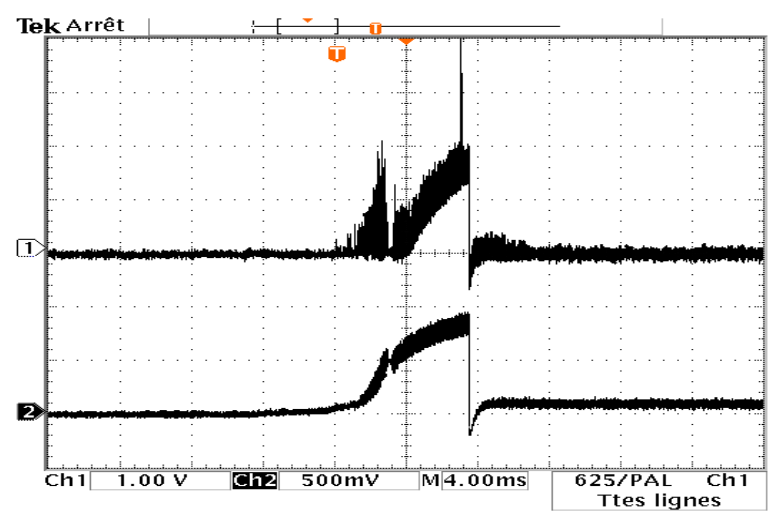

Fig. 10. The simmer voltage (lower trace vertical: $500 \mathrm{~V} / \mathrm{div}$ ) and the voltage pin 4 (upper trace vertical: 1 V/div).

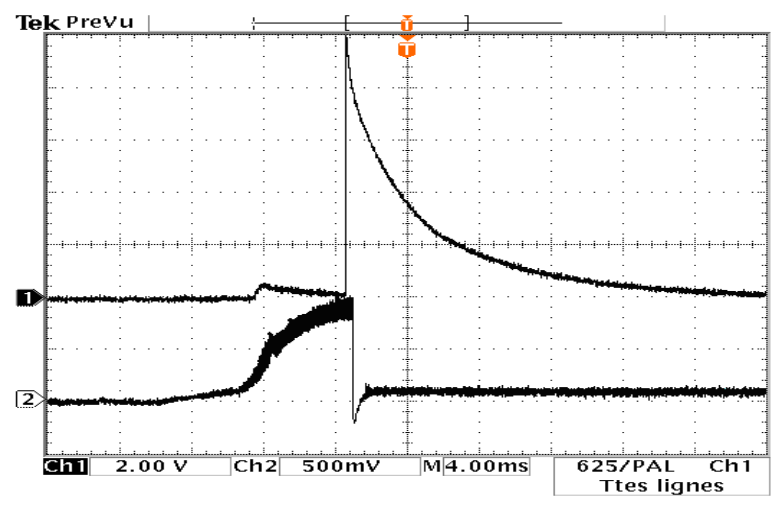

Fig. 11. The trigger pulse (upper trace vertical: $2 \mathrm{kV} / \mathrm{div}$ ) and the simmer voltage (lower trace vertical: $500 \mathrm{~V} / \mathrm{div}$ ). 


\section{Conclusion}

In this article, an automated trigger circuit has been developed in order to overcome the manual triggering when the deionized flash lamp gas can occur. The command circuit is simply implemented using available components allowing a quick maintenance. The experimental results are in good agreement as obtained in simulation.

\section{References}

1. Hook,W.R., Dishington, R.H., Hilberg, R.P.: Xenon Flashlamp Triggering for Laser Applications. IEEE Trans. Electron Device, vol. ED-19, No.3, pp. 308--314, March. (1972)

2. Koechener, W.: Solid-state Laser Engineering. 6th Revised and updated edition, Springer (2006)

3. Changaris, D.G., Louisville, KY (US); Zinner, W.S., New Albany, IN (US): Method and circuit for repetitively firing a flash lamp or the like. United States Patent 6,965,203 B2 (Nov. 15, 2005)

4. Beggar, R., Louhibi, D., Noukaz, A., Almabouada, F.: Caractérisation expérimentale d'une lampe flash utilisée pour le pompage d'un laser à solide. In: the Proceeding SENALAP : Quatrième Séminaire National sur le Laser et ses Applications, pp. 14--17 (2008)

5. Noukaz, A., Louhibi, D., Beggar, R., Almabouada, F.: Modelling And Development Of The Command And Simmer Circuits For The Power Supply Of Flash Lamp Pumped SolidState Lasers. In: AIP Conference Proceedings LAPAMS: First International Conference on Laser and Plasma Applications in Materials Science, pp. 208--211 (2008)

6. Almabouada, F., Louhibi, D., Beggar, R., Noukaz, A., Haddouche, A.: The Main Discharge Transformer And The Trigger Circuit For The Power Supply Of A Flash Lamp-Pumped Solid State Laser. In: AIP Conference Proceedings LAPAMS, pp. 192--195 (2008)

7. Brown, M.: Practical Switching Power Supply Design. Series in Solid State Electronics, Motorola, pp. 29--34 (1991) 